\title{
Pain and Hurt Subscale
}

National Cancer Institute

\section{Source}

National Cancer Institute. Pain and Hurt Subscale. NCI Thesaurus. Code C157700.

A subscale of the Pediatric Quality of Life Brain Tumor Module designed to assess issues associated with pain and hurting. 